\title{
Finding starting points analytically for optical system optimization
}

\author{
F. Bociort* and P. van Grol \\ Delft University of Technology, Optics Research Group \\ Lorentzweg 1, NL - 2628 CJ Delft, The Netherlands
}

\begin{abstract}
Understanding the structure of the design space in optical system optimization is difficult, because common human intuition fails when it encounters the challenge of high dimensionality, resulting from the many optimization parameters of lens systems. However, a deep mathematical idea, that critical points structure the properties of the space around them, is fruitful in lens design as well. Here we discuss simple systems, triplets with curvatures as variables, for which the design space is still simple enough to be studied in detail, but complex enough to be non-trivial. A one-to-one correspondence between the possible design shapes and the critical points resulting from a simplified model based on third-order spherical aberration within the framework of thin-lens theory could lead in the future to a new way to determine good starting points for subsequent local optimization.
\end{abstract}

Keywords: saddle point; critical point; global optimization; optical system design

\section{INTRODUCTION}

While in optical system optimization the focus is usually only on local minima, we have shown recently that other types of critical points (i.e. points where the merit function gradient vanishes) can be very useful as an intermediate step in the process of finding good lens designs. Saddle-point detection (SPD) is a thorough search method that works without any a-priori knowledge for most optimization problems with continuous variables ${ }^{1,2,3}$. The algorithm looks at every known local minimum for index-1 saddle-points around it, and for each detected saddle-point it finds a new minimum on the other side of the "saddle". While SPD is a "blind" and relatively slow search, the algorithm of saddle-point construction (SPC) takes into account special properties of the lens design landscape that allow a fast prediction of the position of saddle points ${ }^{4,5}$. SPC is a tool for changing the system shape in a computationally efficient way that works for optical systems of arbitrary complexity and is presently implemented in the commercial lens design program SYNOPSYS under the name Saddle-point-build (SPB).

For simplicity, here we discuss simple systems, triplets with curvatures as variables, for which the design space is still simple enough to be studied in detail, but complex enough to be non-trivial. All conclusions are also valid in the much simpler problem of doublets. We have shown recently that for these systems a one-to-one correspondence can be found between the possible design shapes and the critical points resulting from a simplified model based on third-order spherical aberration within the framework of thin-lens theory ${ }^{6}$. Here, we develop this idea further, based on new insight resulting from the study of the underlying analytic model ${ }^{7}$.

\section{CRITICAL POINTS}

The critical points can be subdivided depending on their so-called Morse index, which is the number of negative eigenvalues of the Hessian matrix (the matrix of second derivatives of the merit function computed at the given critical point). The Morse index is also the number of downwards directions in the landscape around the critical point. The eigenvalues of local minima are all positive, hence local minima have Morse index 0. Local maxima have Morse index

*email: F.Bociort@tudelft.nl 
equal to the dimension of the design space and saddle points have an index in between these extreme values. In SPD and SPC the emphasis was on saddle points with Morse index 1, that are very similar with the familiar two-dimensional saddle points. However, a deep mathematical idea, that critical points structure the properties of the space around them, suggests that higher indices should not be neglected.

In a study of a thin-film design landscape (that will be discussed in detail elsewhere) it was found that minima form complex, almost random patterns, but critical points with indices higher than 1 form regular patterns that are useful for understanding the topology of the landscape and for finding the minima systematically. For the triplet problem under investigation here, a set of critical points having indices 0, 1 and 2 called fundamental critical points have a remarkable property that shows the existence of order in the design landscape ${ }^{6}$.

\section{CRITICAL - POINT PROJECTION}

Local minima having a similar system drawing and close values of the merit function are considered to have the same "shape". Earlier, we have defined the concept of fundamental design shape ${ }^{6,8}$ and both numerical experiments and analytical models ${ }^{7}$ indicate that for triplets there are 22 fundamental design shapes. We have performed several triplet global searches with different specifications (aperture, field, transverse magnification, etc.) and we have observed that, as long as we have usual glasses and the distances between lenses are not too large (e.g. not much larger than in a Cooke Triplet), any local minimum that is found in such runs can be recognized as having one of the 22 fundamental design shapes. For the specifications used in Ref. 6, the 22 fundamental design shapes are the local minima $\mathbf{M}_{1}-\mathbf{M}_{22}$ shown in Fig.1.
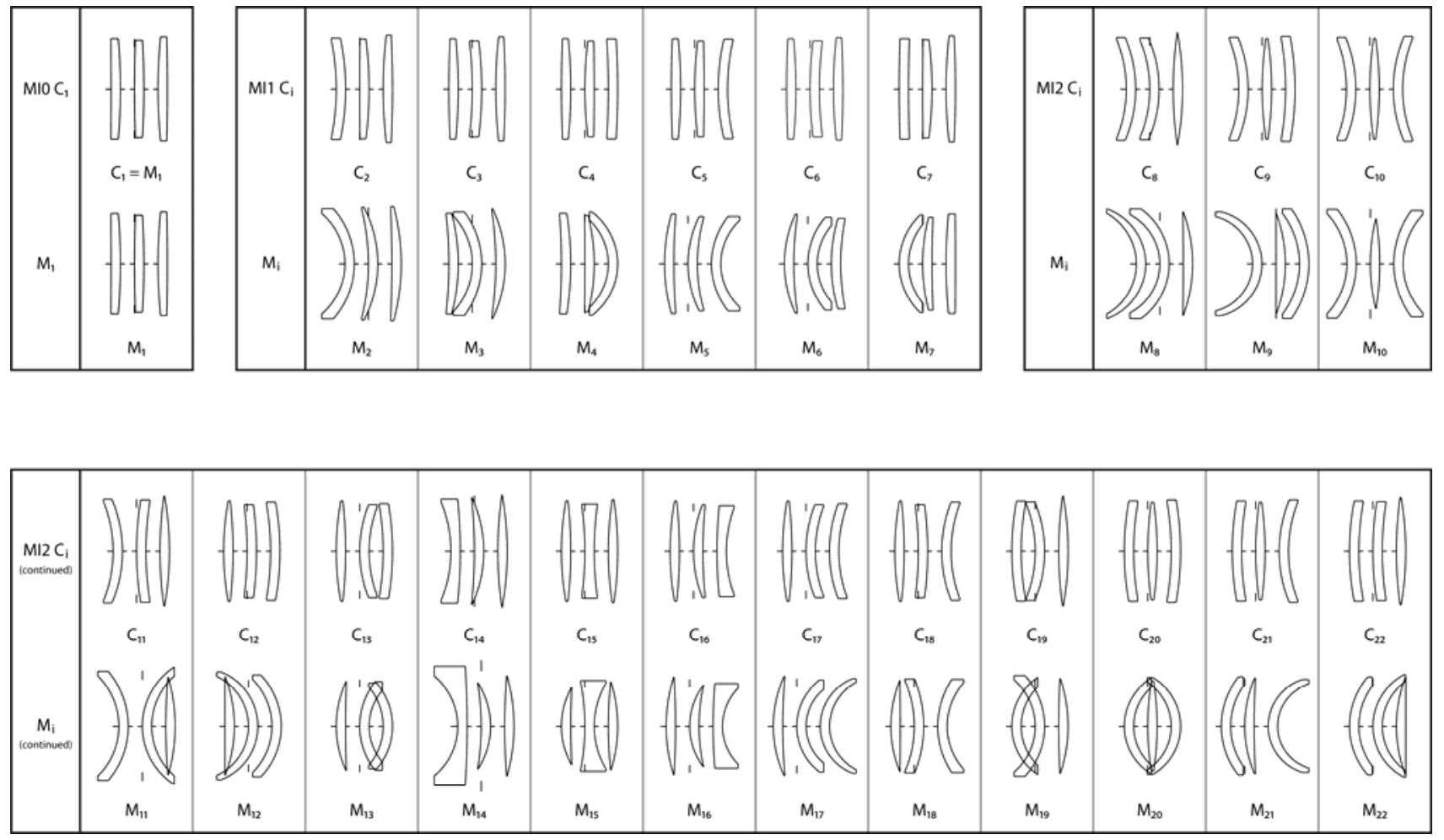

Fig. 1. Fundamental critical points $C_{1}-C_{22}$ and fundamental design shapes $M_{1}-M_{22}$ for triplet global optimization runs. For more details see Ref.6.

Remarkably, there exist 22 critical points, that can be obtained with SPC, one with index 0, 6 with index 1 and 15 with index 2, denoted in Fig 1 by $\mathrm{C}_{1}-\mathrm{C}_{22}$, that strongly resemble the fundamental design shapes. Both sets of systems contain 
meniscus lenses or meniscus-shaped airspaces, and Fig. 1 shows that the main difference between the two types of systems is that the meniscus curvatures are larger in the $M_{i}$ systems than in the corresponding $C_{i}$. Excepting $M_{1}$ (which is identical to $C_{1}$ ) we have a one-to-one correspondence between the $M_{i}$ systems and the corresponding $C_{i}$. We can therefore say that the local minima $\mathrm{M}_{1}-\mathrm{M}_{22}$ are "projections" of the corresponding critical points. The projections can be obtained e.g. via local optimization. Details still need to be polished, but essentially in order to obtain a starting point that leads after optimization to the corresponding $\mathrm{M}_{\mathrm{i}}$ one should simply increase in $\mathrm{C}_{\mathrm{i}}$ the curvatures of the menisci.

In Ref. 6 we have presented two analytical models that can be used to calculate simple approximations for the fundamental critical points of the triplet landscape. When the stop is at the lens, spherical aberration is the most nonlinear of the Seidel aberrations, and determines the non-convex character of the landscape. Both models are based on the third-order spherical aberration of a system of thin lenses in contact, with the stop at the lens. The second model, called "toy model" is especially simple (for mathematical formulas and other details see Refs. 6 and 7). The analytically computed approximations for the critical points can be used instead of the accurate ones computed with SPC for obtaining the fundamental design shapes, even when the numerical merit function used for subsequent local optimization includes all requirements for practical design. In fact, in practical global optimization runs, e.g. in runs having Cooke triplet specifications, the simplest way to obtain all types of local minima for the given specifications (i.e. the design shapes, by ignoring similarly looking solutions with nearly the same merit function) is to do projections as mentioned above, starting from analytically computed fundamental critical points ${ }^{6}$.

While in Ref. 6 the calculation of the curvatures of the fundamental critical points from the variables of the toy model was based on a more elaborate model depending on the marginal ray angles in object and image space, here we use an additional simplification, in order to find the simplest possible description in which the one-to-one correspondence that forms the basis of critical point projection is still recognizable. We now identify the variables $z_{k}$ of the model as surface powers. For triplets, the index $k$ denotes the corresponding surface. The curvature of a surface is then given by

$$
c_{k}=z_{k} /\left(n^{\prime}-n\right)
$$

where $n$ ' is the refractive index after refraction and $n$ is the refractive index before refraction.

For triplets with total power $t$, the 22 fundamental critical points of the toy model are remarkably simple. We collect in the vector $z$ the six variables $z_{k}$. It turns out ${ }^{6,7}$ that there is one critical point with index 0 (i.e. a minimum) given by the vector

$$
z=(t / 6, t / 6, t / 6, t / 6, t / 6, t / 6)
$$

Depending on the position $k$ where the minus sign is placed, there are 6 critical points with index 1 , e.g. for minus sign at $k=1$ we have

$$
z=(-t / 4, t / 4, t / 4, t / 4, t / 4, t / 4)
$$

By permuting two minus signs over six positions, we find $6 * 5 / 2=15$ critical points with index 2 , e.g.

$$
z=(t / 2, t / 2,-t / 2,-t / 2, t / 2, t / 2)
$$

By using in Eq. (1) for all three lenses $n=1.5$, we obtain for the fundamental critical points the drawings shown in Fig. 2. Note the resemblance of these systems with critical points $C_{1}-C_{22}$ shown in Fig. 1. Those interested in philosophy may be tempted to identify the systems shown in Fig. 2 with Platonic "Forms" that capture the essence of the manifested phenomena, which are in this case local minima such as $\mathrm{M}_{1}-\mathrm{M}_{22}$ in Fig. 1, for which the exact details change, depending on the exact specifications (aperture, field, magnification etc.).

To understand critical-point projection, it is useful to examine the simplest case when the one-to-one correspondence between fundamental critical points and design shapes occurs. This is the case of the toy model for a doublet with the first surface curvature constant and the other three variable, subject to the constraint that the total power is constant. It turns out that in this case we have four fundamental critical points, one minimum (called main hub) and three saddle points with index $1, C_{2}-C_{4}$, and four local minima (the design shapes $M_{1}-M_{4}$ ), one of them being the main hub $M_{1}=C_{1}$. Because we have now only two independent variables (for details see Eqs. 16 in Ref. 7) all these points can be plotted in a plane. Critical point projection can be observed in Fig. 3: the three minima $M_{2}-M_{4}$ are placed on straight lines defined by the main hub $\mathrm{M}_{1}=\mathrm{C}_{1}$ and the saddle points $\mathrm{C}_{2}-\mathrm{C}_{4}$. 


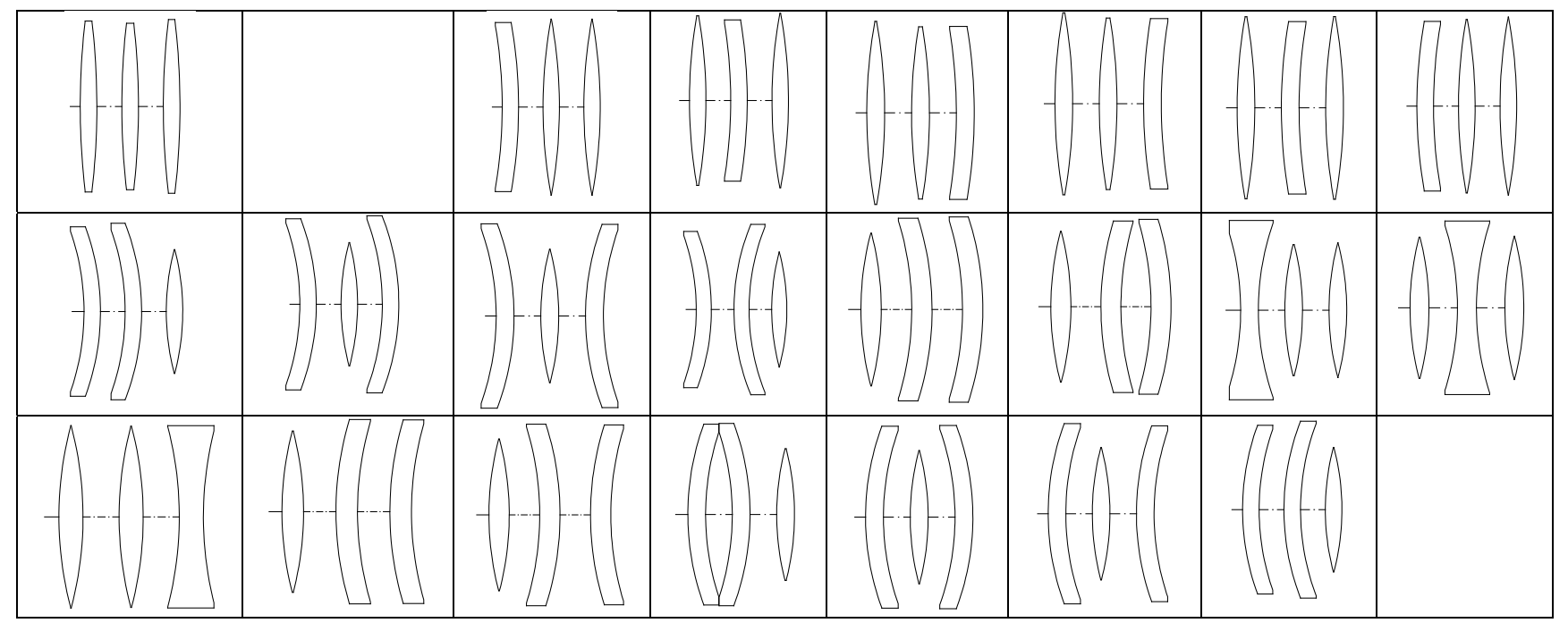

Fig. 2. The fundamental critical points of the toy model, when the variables of the model are considered to be the surface powers. The first row shows the index 0 system $C_{1}$ and the six index 1 systems $C_{2}-C_{7}$. Rows 2 and 3 show the 15 index 2 solutions $C_{8}$ - $C_{22}$.

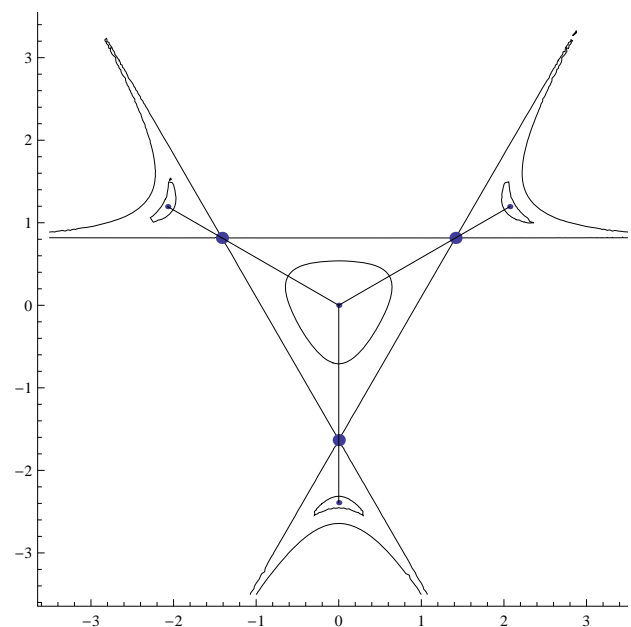

Fig.3 Equimagnitude contours of the toy model with three variables and one constraint. Local minima $\left(\mathrm{M}_{1}-\mathrm{M}_{4}\right)$ are shown with small dots, saddle points $\left(\mathrm{C}_{2}-\mathrm{C}_{4}\right.$, situated there where two equimagnitute lines cross) are shown with large dots.

In fact, the toy model for triplets with six variables and one constraint turns out to have exactly the same property: all minima $\mathrm{M}_{2}-\mathrm{M}_{22}$ are located on straight lines defined by the main hub $\mathrm{M}_{1}=\mathrm{C}_{1}$ and the saddle points $\mathrm{C}_{2}-\mathrm{C}_{22}$. Since these saddle points are known, finding the design shapes can be done simply with a one-dimensional search along these lines.

The toy model described in Refs. 6 and 7 simulates only third-order spherical aberration. It turns out that when in this model we add an additional term that simulates third-order coma (details will be given elsewhere), this beautiful symmetry is destroyed and the minima are not placed exactly on these lines any more. However, it turns out that the number of fundamental critical points and system shapes remains unchanged and that the one-to one correspondence is still valid. Critical-point projection can be implemented by numerically optimizing starting points chosen on the lines mentioned above, close to the critical points, but on the side opposite to the main hub. 


\section{CONCLUSIONS}

The purpose of this research is twofold. First, the one-to-one correspondence between the remarkably simple fundamental design shapes of the toy model for triplets in Fig. 2 given by Eqs. (1-4) and the set of all possible types of minima (i.e. the fundamental critical points) of a numerical merit function including all requirements for practical design shows an unsuspected degree of order in the design landscape, resulting from the deep mathematical property that critical points (including those with index higher than 1) structure the space around them.

On the other hand, when we have triplets with usual glasses and the distances between lenses are not too large, criticalpoint projection can be used to obtain all design shapes existing in the landscape (a subset of those shown in Fig. 1) from starting points computed analytically. We plan to research whether it is possible to extend this approach to complex systems where analytical critical-point projection is applied to different groups of three neighboring lenses. Several versions of the analytical model have been developed in order to examine which one will give the best results when applied for complex systems.

\section{ACKNOWLEDGMENTS}

The use of a CODE V educational license is gratefully acknowledged.

\section{REFERENCES}

[1] Bociort, F., Van Driel, E. and Serebriakov, A., "Networks of local minima in optical system optimization,” Optics Letters 29, 189-191 (2004).

[2] Van Driel, E., Bociort, F. and Serebriakov, A., ’Topography of the merit function landscape in optical system design,” Proc. SPIE 5249, 353-363 (2004).

[3] Marinescu, O. and Bociort, F., "Network search method in the design of EUV lithographic objectives,” Applied Optics 46, 8385-8393 (2007).

[4] Bociort, F., and Van Turnhout, M., "Finding new local minima in lens design landscapes by constructing saddle points”, Optical Engineering 48, 063001 (2009).

[5] Van Turnhout, M., Bociort, F. and Van Grol, P., "Systematically obtaining new local minima via the Saddle-Point Construction method," to be published.

[6] Bociort, F. and Van Grol, P., "Systematics of the design shapes in the optical merit function landscape," Proc. SPIE 7717, 77170 (2010).

[7] Bociort, F., “Why are there so many system shapes in lens design?”, Proc. SPIE 7849, 78490D (2010)

[8] Van Grol, P., Bociort, F. and Van Turnhout, M., "Finding order in the design landscape of simple optical systems", Proc. SPIE 7428, 742808, (2009). 